\title{
TRATAMENTO DE EFLUENTES CONTENDO VERDE MALAQUITA UTILIZANDO PALHA DE MILHO MODIFICADA VIA ULTRASSOM
}

\author{
L. KLEIN ${ }^{1}$, D. R. LIMA ${ }^{1}$ e G. L. DOTTO ${ }^{1}$ \\ ${ }^{1}$ Universidade Federal de Santa Maria, Departamento de Engenharia Química. \\ E-mail para contato: laura_klein83@hotmail.com
}

\begin{abstract}
RESUMO - O presente trabalho teve como objetivo estudar a remoção do corante verde malaquita de águas residuais através da adsorção utilizando palha de milho in natura e tratada via ultrassom. O modelo de pseudo-segunda ordem foi adequado para a cinética de adsorção e o modelo de Freundlich foi apropriado para representar o equilíbrio. A máxima capacidade de adsorção encontrada para os experimentos com efluente sintetizado em concentração de $200 \mathrm{mg} \mathrm{L}^{-1}$ foi de $257,1 \mathrm{mg} \mathrm{g}^{-1}$ para a palha de milho in natura e de $283,8 \mathrm{mg} \mathrm{g}^{-1}$ para o adsorvente modificado, gerando $66,6 \%$ e 73,3\% de remoção, respectivamente, enquanto para os experimentos com o efluente real obteve-se $56,3 \%$ e $63,2 \%$, respectivamente.
\end{abstract}

\section{INTRODUÇÃO}

Com o desenvolvimento industrial, agravam-se os problemas ambientais devido à eliminação de rejeitos tóxicos. Efluentes industriais contendo corantes sintéticos são gerados em diferentes setores industriais, tais como têxtil, papel, couro, plástico e processamento mineral, e o seu despejo é uma das principais fontes de poluição aquática (Khataee et al., 2015). Esses rejeitos, quando eliminados sem um tratamento adequado, representam perigo ao meio ambiente (Srinivasan e Viraraghavan, 2010). Existem diversos métodos de tratamento para a remoção de corantes de efluentes aquosos. Uma das técnicas mais eficientes é a adsorção, devido ao seu baixo custo, baixa necessidade energética e simples operação (Esquerdo et al., 2014). Devido à necessidade de um adsorvente presente em larga escala, reutilizável e de baixo custo, resíduos agroindustriais vêm sido pesquisados na remoção de corantes. Neste trabalho, objetivou-se estudar a adsorção do corante Verde Malaquita, utilizando palha de milho in natura e modificada via ultrassom.

\section{MATERIAL E MÉTODOS}

\subsection{Desenvolvimento do adsorvente}

A palha de milho foi obtida em uma propriedade rural no estado do Rio Grande do Sul. $\mathrm{O}$ material passou por lavagem com água destilada, secagem em estufa por $24 \mathrm{~h}$ a $40{ }^{\circ} \mathrm{C}$, moagem e peneiramento até atingir a faixa granulométrica de 68 a $75 \mu \mathrm{m}$. Em seguida, o pó resultante foi lavado novamente com água deionizada, até atingir $\mathrm{pH} 7$, e seco em estufa por 24 $\mathrm{h}$ a $40{ }^{\circ} \mathrm{C}$. A modificação via ultrassom da palha de milho realizou-se em um processador ultrassônico (UP400S, Hielscher, Alemanha) de 400 W, equipado com um sonotrodo de titânio, 
durante $1 \mathrm{~h}$ a $24 \mathrm{kHz}$. Os parâmetros utilizados foram: ciclo de 1, amplitude de 90\% e temperatura de $25{ }^{\circ} \mathrm{C}$. Após o tratamento, a palha de milho foi separada por filtração e sua secagem foi realizada em estufa. Os adsorventes in natura e modificado via ultrassom foram caracterizados via microscopia eletrônica de varredura (MEV) (Jeol, JSM-6610LV, Japão) e espectroscopia em infravermelho por transformada de Fourier (FT-IR) (Prestige, 21210045, Japão) e ponto de carga zero ( $\mathrm{pH}_{\mathrm{PCZ}}$ ).

\subsection{Ensaios de adsorção}

Para o preparo das soluções sintéticas, utilizou-se o corante verde malaquita $\left(\mathrm{C}_{23} \mathrm{H}_{25} \mathrm{ClN}_{2}\right.$, massa molecular de 364,9 $\mathrm{g} \mathrm{mol}^{-1}$, pureza de 98\%), fornecido pela Synth, Brasil. O efluente real, gerado através de descartes laboratoriais, consiste em uma mistura de corantes. Entre eles estão o verde malaquita, cristal violeta e azul de metileno, em concentração desconhecida. Todos os testes de adsorção foram realizados em batelada em um agitador termostatizado (Marconi, MA 093, Brasil), com agitação constante de $200 \mathrm{rpm}$. O volume de solução foi fixado em $50 \mathrm{~mL}$, a massa de adsorvente em $0,025 \mathrm{~g}$ e a temperatura em $25^{\circ} \mathrm{C}$. O pH foi ajustado para 6. Para o estudo cinético, utilizou-se uma solução de verde malaquita com concentração de 25 $\mathrm{mg} \mathrm{L}^{-1}$ variando-se o tempo de contato em 2, 6, 10, 20, 30, 60, 90, 120 e 240 minutos. Para a construção das isotermas de adsorção, o adsorvente ficou em contato com a solução por $4 \mathrm{~h}$, variando-se a concentração inicial do adsorbato em 25, 50, 100 e $200 \mathrm{mg} \mathrm{L}^{-1}$.Após estes testes, amostras foram coletadas e centrifugadas (Centribio, 80-131 2B, Brasil) a $5000 \mathrm{rpm}$ por 30 minutos, e a concentração remanescente de verde malaquita na fase aquosa foi determinada por espectrofotometria em $K=617 \mathrm{~nm}$ (Biospectro SP-22, Brasil). Com os resultados das análises, calculou-se a capacidade de adsorção no equilíbrio $\left(\mathrm{q}_{\mathrm{e}}\right)$ de cada amostra, conforme a equação 1 .

$$
q_{e}=\frac{\left(C_{0}-C_{e}\right)}{m}
$$

O experimento de adsorção com o resíduo de corantes gerado em laboratório foi realizado com um tempo de contato de $4 \mathrm{~h}$ e após encaminhado para varredura espectral (SHIMADZU, UV-2600). Através do cálculo da área abaixo das curvas geradas antes e após adsorção e aplicação na equação 2, obteve-se o percentual de remoção:

$$
R=\frac{A_{R}-A_{B}}{A_{R}} 100 \%
$$

\subsection{Cinética e isotermas}

Os modelos cinéticos de pseudo-primeira ordem, representado pela equação 3, e pseudosegunda ordem, conforme a equação 4, foram usados para representar a cinética de adsorção.

$$
q_{e}=q_{1}\left(1-\exp \left(-k_{1} t\right)\right)
$$




$$
q_{e}=\frac{t}{\frac{1}{k_{2} q_{2}{ }^{2}}+\frac{t}{q_{2}}}
$$

Os dados experimentais obtidos no estudo do equilíbrio foram ajustados aos modelos de isotermas de Langmuir e de Freundlich, representados pelas equações 5 e 6, respectivamente.

$$
\begin{aligned}
& q_{e}=\frac{q_{m} k_{L} C_{e}}{1+k_{L} C_{e}} \\
& q_{e}=k_{F} C_{e}^{1 / n_{F}}
\end{aligned}
$$

\section{RESULTADOS E DISCUSSÃO}

\subsection{Caracterização do adsorvente}

O FT-IR dos adsorventes apresentou similaridade, indicando que não houve degradação dos grupos funcionais devido ao tratamento ultrassônico. Os principais grupos funcionais identificados foram hidroxila, carboxila e lactonas. As imagens obtidas via MEV estão apresentadas nas Figuras 1A e 1B. Observa-se que a palha de milho in natura (2A) possui uma estrutura não-porosa, enquanto que o adsorvente com tratamento ultrassônico (2B) apresentou cavidades e protuberâncias. Isto aponta que a modificação via ultrassom foi adequada para alterar as características estruturais da palha de milho. O ponto de carga zero correspondeu ao $\mathrm{pH} 2,3$, mostrando que o corante verde malaquita é propício a ser adsorvido pela palha de milho.

Figura 1: Microscopia eletrônica de varredura para (A) palha de milho in natura (B)Modificada via ultrassom.

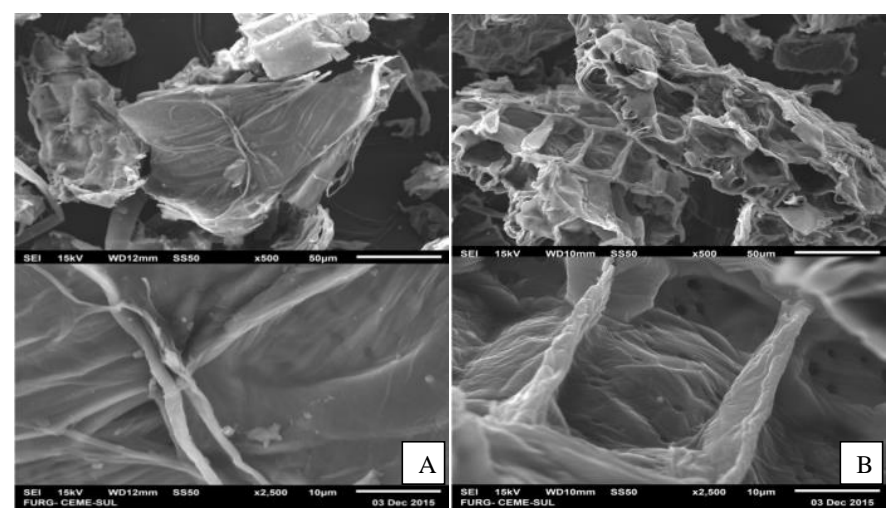

\subsection{Estudo cinético e de equilíbrio}

A Figura 2 apresenta a capacidade de adsorção de verde malaquita, para o adsorvente in natura e modificado, em função do tempo. 
Figura 2: Cinética da adsorção de verde malaquita em palha de milho in natura e modificada via ultrassom $\left(\mathrm{T}=25^{\circ} \mathrm{C}, 0,025 \mathrm{~g}\right.$ de adsorvente, $\left.\mathrm{C}_{0}=25 \mathrm{mg} \mathrm{L}^{-1}, \mathrm{pH} 6\right)$.

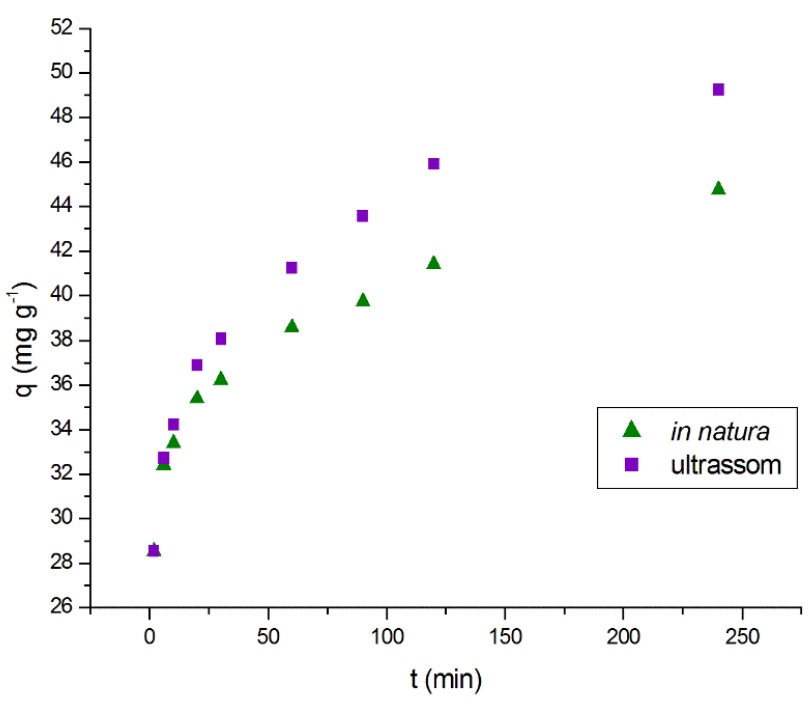

Os modelos de pseudo-primeira ordem e pseudo-segunda ordem foram ajustados aos dados experimentais. Os parâmetros obtidos nos ajustes estão apresentados na Tabela 1 e 2. O modelo que melhor descreveu o comportamento cinético do processo foi o de pseudo-segunda ordem, tanto para a palha de milho in natura, como para o adsorvente com tratamento ultrassônico. A capacidade teórica de adsorção obtida no modelo foi de $39,9 \mathrm{mg} \mathrm{g}^{-1}$ para o adsorvente in natura, e de 43,55 $\mathrm{mg} \mathrm{g}^{-1}$ para o adsorvente modificado.

Tabela 1: Parâmetros cinéticos

\begin{tabular}{ccc}
\hline & $\begin{array}{c}\text { Pseudo-primeira ordem } \\
\text { in natura }\end{array}$ & Modificado \\
\hline $\mathrm{q}_{1}\left(\mathrm{mg} \mathrm{g}^{-1}\right)$ & 37,9 & 40,8 \\
$\mathrm{k}_{1}\left(\mathrm{~min}^{-1}\right)$ & 0,633 & 0,484 \\
$\mathrm{R}^{2}$ & 0,9582 & 0,9363 \\
$\mathrm{EMR}(\%)$ & 7,40 & 10,09 \\
\hline & Pseudo-segunda ordem & \\
Parâmetro & in natura & Modificado \\
\hline $\mathrm{q}_{2}\left(\mathrm{mg} \mathrm{g}^{-1}\right)$ & 39,8 & 43,5 \\
$\mathrm{k}_{2} \times 10^{3}\left(\mathrm{~g} \mathrm{mg}^{-1} \mathrm{~min}^{-1}\right)$ & 0,0233 & 0,0145 \\
$\mathrm{R}^{2}$ & 0,9798 & 0,9699 \\
$\mathrm{EMR} \%)$ & 5,28 & 7,13 \\
\hline
\end{tabular}

Os dados experimentais obtidos no estudo do equilíbrio estão apresentados na Figura 3. A máxima capacidade experimental de adsorção foi, para uma concentração inicial de $200 \mathrm{mg}$ $\mathrm{L}^{-1}$, de $257,10 \mathrm{mg} \mathrm{g}^{-1}$ para a palha de milho in natura e de $283,81 \mathrm{mg} \mathrm{g}^{-1}$ para a palha de milho modificada, com $66,64 \%$ e 73,32 \% de remoção, respectivamente. Isto indica uma melhoria 
nas características estruturais do adsorvente causada pelo tratamento ultrassônico. Os modelos de Freundlich e Langmuir foram ajustados aos dados experimentais, estando os parâmetros apresentados na tabela 2. O modelo que melhor representou o equilíbrio de adsorção, para o adsorvente in natura e também com tratamento ultrassônico, foi a isoterma de Freundlich, devido aos maiores valores de $\mathrm{R}^{2}$ e menores valores do erro médio padrão.

Figura 3: Isotermas de adsorção de verde malaquita em palha de milho in natura e modificada via ultrassom.

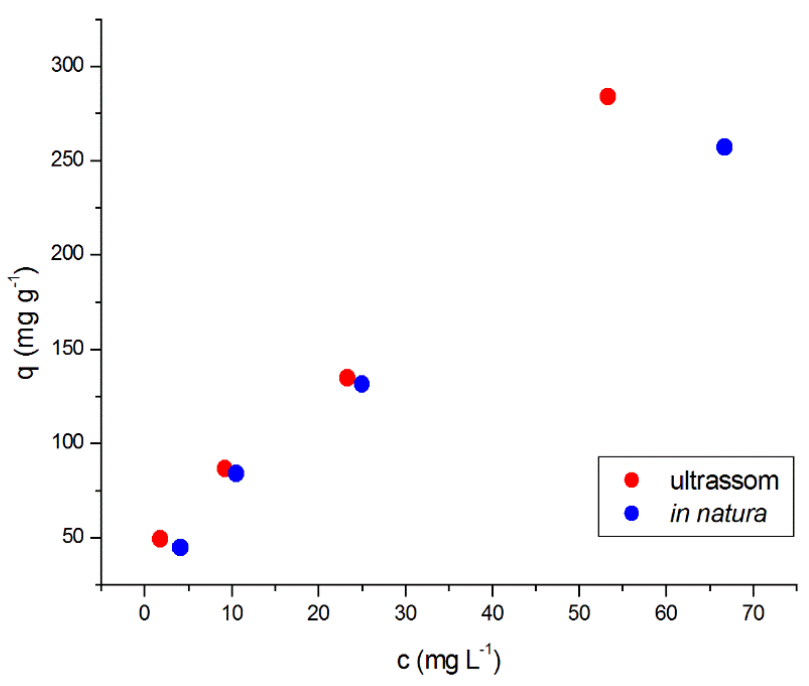

Tabela 2: Parâmetros de equilíbrio

\begin{tabular}{ccc}
\hline & $\begin{array}{c}\text { Modelo de Freundlich } \\
\text { in natura }\end{array}$ & Modificado \\
\hline$k_{F}\left(\left(\mathrm{mg} \mathrm{g}^{-1}\right)\left(\mathrm{mg} \mathrm{L}^{-1}\right)\right)^{\mathrm{n}} \mathrm{F}$ & 17,93 & 17,77 \\
$1 / n_{F}$ & 0,63 & 0,69 \\
$\mathrm{R}^{2}$ & 0,9992 & 0,9892 \\
$\mathrm{EMR}(\%)$ & 2,47 & 13,70 \\
\hline Parâmetro & Modelo de Langmuir & \\
\hline$q_{m}\left(\mathrm{mg} \mathrm{g}^{-1}\right)$ & in natura & Modificado \\
$\left.k_{L}(\mathrm{~L} \mathrm{mg})^{-1}\right)$ & 460,14 & 737,97 \\
$\mathrm{R}^{2}$ & 0,0184 & 0,0114 \\
$\left.\mathrm{EMR}^{2} \%\right)$ & 0,9944 & 0,9801 \\
& 10,04 & 20,96 \\
\hline
\end{tabular}

\subsection{Estudo com efluente gerado em laboratório}

O resultado dos ensaios com o efluente gerado em laboratório está ilustrado na figura 4. A remoção percentual foi calculada através da equação 2 , a partir do valor das áreas abaixo das bandas de adsorção. Obtiveram-se 56,34 \% de remoção para a palha de milho in natura e 
$63,16 \%$ para o adsorvente modificado. $\mathrm{O}$ adsorvente mostrou-se, portanto, adequado para o tratamento desde efluente.

\section{CONCLUSÃO}

O presente estudo mostrou que a palha de milho pode ser empregada como um adsorvente promissor na remoção do corante verde malaquita de soluções aquosas, assim como no tratamento de efluentes contendo diversos corantes. A cinética foi melhor representada pelo modelo de pseudo-segunda ordem e o equilíbrio foi descrito mais adequadamente pela isoterma de Freundlich. As capacidades de adsorção foram de $257,1 \mathrm{mg} \mathrm{g}^{-1}$ para a palha de milho in natura e de $283,8 \mathrm{mg} \mathrm{g}^{-1}$ para a palha de milho modificada. $\mathrm{O}$ adsorvente também se mostrou adequado para o tratamento de efluentes contendo misturas de corantes em solução aquosa.

Figura 4: Varredura espectral do resíduo sem tratamento e após o processo de adsorção com os adsorventes in natura e modificado.

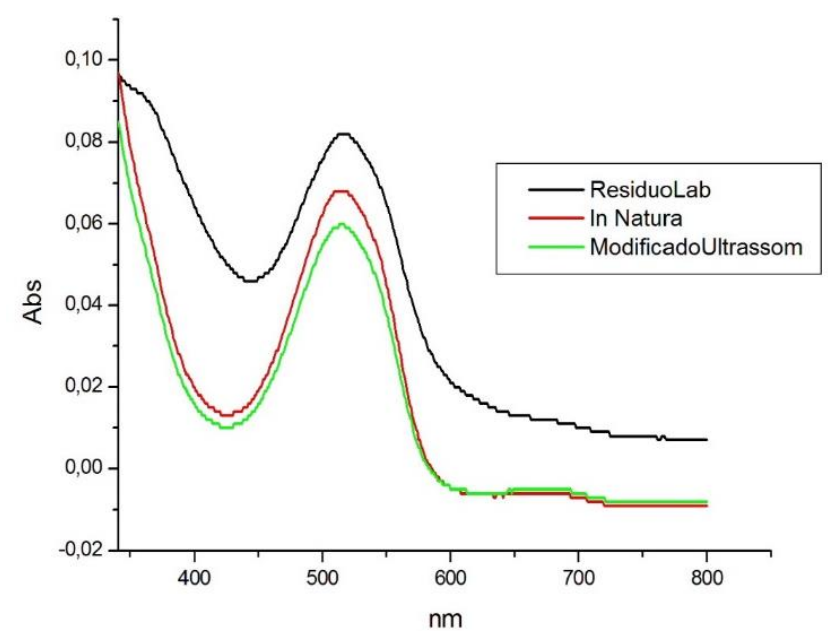

\section{REFERÊNCIAS}

ESQUERDO, T. R. S.; CADAVAL, G. L.; DOTTO, G. L.; PINTO, L. A. A. Chitosan scaffold as an alternative adsorbent for the removal of hazardous food dyes from aqueous solutions. Colloid Interface Sci. v. 424, p. 7-15, 2014.

KHATAEE, A.; SHEYDAEI, M.; HASSANI, A.; TASEIDIFAR, M. KARACA, S.

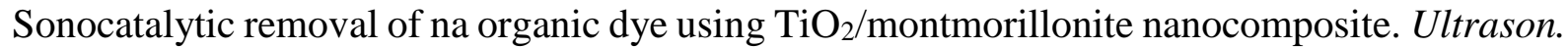
Sonochem. v. 22, p. 404-411, 2015.

SILVA, F. M.; SANTANA, S. A. A.; BEZERRA, C. W. B; SILVA, H. A. S.; Adsorção do Corante Têxtil Azul de Remazol R por Pseudocaule da Bananeira (Musa sp). Cad. Pesq., v. 17 n. 3, p. 71-77, 2010

SRINIVASAN, A.; VIRARAGHAVAN, T. Decolorization of dye wastewaters by biosorbents: A review. J. Environ. Manage. v. 91, p. 1915-1929, 2010.

ZHANG, P.; WANG, T.; QIAN, G.; WU, D; FROST, R. L. Removal of methyl orange from aqueous solutions through adsorption by calcium aluminate hydrates. Colloid Interface Sci. v. 426, p. 44-47, 2014. 